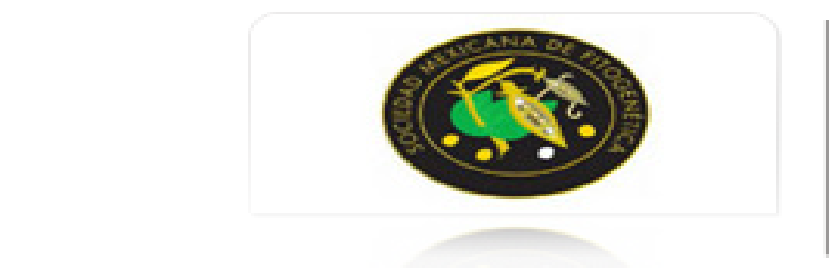

Revista Fitotecnia Mexicana

ISSN: 0187-7380

revfitotecniamex@gmail.com

Sociedad Mexicana de Fitogenética, A.C.

México

Fuentes-Dávila, Guillermo; Figueroa-López, Pedro; Camacho-Casas, M. Alfonso; Chávez-Villalba,

Gabriela; Félix-Fuentes, José L.

'QUETCHEHUECA ORO C2013', NUEVA VARIEDAD DE TRIGO CRISTALINO PARA EL NOROESTE DE MÉXICO

Revista Fitotecnia Mexicana, vol. 37, núm. 4, 2014, pp. 399-401

Sociedad Mexicana de Fitogenética, A.C.

Chapingo, México

Disponible en: http://www.redalyc.org/articulo.oa?id=61032672013

- Cómo citar el artículo

- Número completo

- Más información del artículo

Página de la revista en redalyc.org

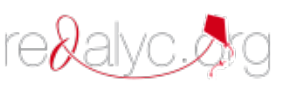

Sistema de Información Científica

Red de Revistas Científicas de América Latina, el Caribe, España y Portugal

Proyecto académico sin fines de lucro, desarrollado bajo la iniciativa de acceso abierto 


\title{
'QUETCHEHUECA ORO C2013', NUEVA VARIEDAD DE TRIGO CRISTALINO PARA EL NOROESTE DE MÉXICO
}

\author{
'QUETCHEHUECA ORO C2013', A NEW DURUM WHEAT \\ CULTIVAR FOR NORTHWEST MÉXICO
}

\section{Guillermo Fuentes-Dávila*, Pedro Figueroa-López, M. Alfonso Camacho-Casas, Gabriela Chávez-Villalba y José L. Félix-Fuentes}

\begin{abstract}
Campo Experimental Norman E. Borlaug, Centro de Investigación Regional del Noroeste, Instituto Nacional de Investigaciones Forestales, Agrícolas y Pecuarias. Km. 12 Norman E. Borlaug, Apdo. Postal 155. 85000, Cd. Obregón, Sonora. Tel. 01 (644) 4145700 Ext. 254.

*Autor para correspondencia: (fuentes.guillermo@inifap.gob.mx)
\end{abstract}

La producción mundial de trigo (Triticum aestivum L.) en el año 2010 fue de 651.9 millones de toneladas (FAOSTAT, 2011), y los principales productores fueron China, India, Estados Unidos, la Federación Rusa y Francia. México produjo 3.6 millones de toneladas, de las cuales exportó 1.1 millones de toneladas de trigo cristalino (OEIDRUS, 2013), pero importó 3.5 millones de toneladas de trigo harinero. Entre los sectores agroindustriales alimenticios en México, el trigo ocupa el segundo lugar en importancia después del maíz (Zea mays L.) (CANIMOLT, 2011). En este país, la superficie total sembrada con trigo en el ciclo otoño-invierno 2009-2010 fue 866,000 ha (INEGI, 2010), y en el Estado de Sonora se sembraron 247,281 ha $(28.55 \%)$.

El trigo en Sonora es de hábito de crecimiento de primavera y se cultiva en condiciones de riego. En México, el trigo duro o cristalino se produce principalmente en este estado, donde a partir del ciclo 2001-2002, de las 190,718 ha sembradas el trigo cristalino ocupó poco más de $70 \%$ de esa superficie (Camacho-Casas et al., 2004). La preferencia por esta clase de trigo se debe a su alto potencial de rendimiento, aceptación en el mercado de exportación, su tolerancia al carbón parcial (Tilletia indica Mitra) y a la roya de la hoja (Puccinia triticina Eriksson). La variedad de trigo cristalino más sembrada en la región, hasta el ciclo 2002-2003, fue 'Altar C84', a pesar que su resistencia a la roya de la hoja ya había sido vencida por la raza BBG-BN (Singh et al., 2004), que disminuyó su producción en los ciclos 2000-2001 y 2001-2002 (Figueroa-López et al., 2002; Com. Pers. ${ }^{1}$ ).

La variedad 'Júpare C2001' (Camacho-Casas et al., 2004) dominó el área dedicada al trigo en el sur de Sonora desde el ciclo 2003-2004 hasta el 2008-2009, donde se sembró en

${ }^{1}$ Figueroa-López P., L. A. Gaxiola-Verdugo, A. Suárez-Beltrán, R. Álvarez-Zamorano y M. A. Camacho-Casas (2002) Análisis comparativo de las epidemias de roya de la hoja de trigo cristalino en el Valle del Yaqui, Sonora, México, en los años 2001 y 2002. Memorias XXIX/V Congreso Nacional/Internacional de la Soci. Mex. de Fitopatología. Julio 2 al 5 de 2002, Monterrey, Nuevo León, México. Resumen F-73.
119,327 ha (42.3\%), pero su resistencia también sucumbió ante otra nueva raza de roya (BBG-PN). La variedad 'CIRNO C2008', liberada en el año 2008, ha demostrado su resistencia a la roya de la hoja gracias a la contribución del progenitor CAMAYO, el cual posee un gen de resistencia ausente en todas las demás variedades comerciales, y ocupó 87,105 ha en el ciclo agrícola 2010-2011 en el sur de Sonora, 154,915 en el ciclo 2011-2012 y 196,295 en el 2012-2013. Este incremento sustancial en área sembrada se ha debido a su alto potencial de rendimiento; sin embargo, en el último ciclo mostró una reacción de moderadamente resistente a moderadamente susceptible a la roya lineal o amarilla (Puccinia striiformisf. sp. tritici).

Por esto es importante ampliar las opciones en el mosaico genético de variedades de trigo recomendadas, así como contribuir a incrementar la vida útil de las variedades que los productores de Sonora y del noroeste de México siembran, y a la vez cumplir con los requerimientos mínimos de calidad para el mercado de la exportación. Como resultado de los trabajos de mejoramiento y de las evaluaciones del rendimiento de grano llevadas a cabo a partir del ciclo otoño-invierno 2009-2010 en el Campo Experimental Norman E. Borlaug (CENEB), perteneciente al Instituto Nacional de Investigaciones Forestales, Agrícolas y Pecuarias, se propuso la liberación de la variedad de trigo cristalino 'Quetchehueca Oro C2013'.

En este escrito se aportan las comparaciones de rendimiento y los parámetros de calidad durante los ciclos agrícolas 2011-2012 y 2012-2013 con respecto al testigo 'CIRNO C2008', variedad de trigo cristalino más cultivada en Sonora. 'Quetchehueca Oro C2013' representa una nueva opción de materiales de trigo cristalino con un potencial de rendimiento aceptable y una mejor calidad para la elaboración de pastas, y para su producción por los agricultores de Sonora y del noroeste de México interesados en la exportación de grano. 
Esta nueva variedad se obtuvo por hibridación y selección a través del método masal modificado, a partir de la cruza GODRIN/GUTROS//DUKEM/3/THKNEE_11/4/ DUKEM_1//PATKA_7/YAZI_1/3/PATKA_7/YAZI_1/5/ AJAIA_12/F3LOCAL(SEL.ETHIO.135.85)//PLATA_13/3/ ADAMAR, llevada a cabo por el Centro Internacional de Mejoramiento de Maíz y Trigo (CIMMYT).

Su número de cruza e historial de selección es CDSS04B00367T-0TOPY-10Y-0M-4Y-0M-4Y-0B. Las selecciones individuales y en masa se efectuaron alternadamente en las estaciones experimentales de El Batán, Edo. de México (B) (19³0’ N, 2249 msnm), San Antonio Atizapán, Edo. de México (M) (19 $\left.17^{\circ} \mathrm{N}, 2640 \mathrm{msnm}\right)$ y en el CENEB en el Valle del Yaqui (Y) (27²0’ N, 40 msnm), en Sonora. Las condiciones de humedad fueron de secano (temporal) regular en las estaciones del Centro de México y riego normal en el Valle del Yaqui.

'Quetchehueca Oro C2013' cuenta con el registro definitivo TRI-150-260913 del Catálogo Nacional de Variedades Vegetales (CNVV) del Servicio Nacional de Inspección y Certificación de Semillas (SNICS). Sus evaluaciones de rendimiento y calidad industrial se llevaron a cabo en los ciclos agrícolas 2009-2010 a 2011-2012 en el CENEB. El rendimiento experimental de grano que se obtuvo en varias fechas de siembra (5.87 t ha-1), es $155 \mathrm{~kg}$ superior al testigo 'CIRNO C2008'. Las mejores fechas para sembrar esta variedad son entre el 15 de noviembre y el 1 de diciembre, pues su rendimiento disminuye significativamente en las siembras tardías del 15 de diciembre al 1 de enero.

Durante el ciclo agrícola 2011-2012, en un lote de validación con un agricultor cooperante en la manzana 1803 del Valle del Yaqui, 'Quetchehueca Oro C2013' rindió hasta $10.5 \mathrm{t} \mathrm{ha}^{-1}$. Su altura promedio es de $83 \mathrm{~cm}$, con ciclo de 82 d a espigamiento y 125 d a madurez fisiológica. Presenta una coloración de antocianina fuerte en el coleóptilo y muy baja o nula frecuencia de plantas con hoja bandera curvada. La espiga tiene un perfil de bordes paralelos, densidad densa y longitud mediana $(7.0$ a $8.5 \mathrm{~cm})$; produce de 20 a 22 espiguillas, y cada espiguilla produce de 1 a 5 granos en el tercio inferior, de 2 a 5 en el tercio medio de la espiga, y de 1 a 4 en el tercio superior. Su planta, espiga y grano se ilustran en la Figura 1.

En el tercio medio de la espiga el hombro de la gluma es angosto y de forma inclinada, con una punta corta de forma moderadamente curva sin vellosidad en la superficie externa. El grano es de color ámbar, semi-elíptico, de 7.4 $\mathrm{mm}$ de longitud, $3.6 \mathrm{~mm}$ de ancho, con peso medio de 64 $\mathrm{mg}$, y produce entre 10,751 y 14,100 granos $/ \mathrm{m}^{2}$. El color del grano puede ser muy tenue o estar ausente al ser tratado con fenol. El peso específico medio es de $81.3 \mathrm{~kg} \mathrm{hL}^{-1}$.

'Quetchehueca Oro C2013' y ‘CIRNO C2008' presentan resistencia a la roya de la hoja, pero la segunda ha mostrado una reacción de moderadamente resistente a moderadamente susceptible a la roya amarilla o lineal, mientras que la primera es resistente. Ambas variedades presentan resistencia al carbón parcial. 'Quetchehueca Oro C2013' contiene un promedio de $12.1 \%$ de proteína en grano y 28.7 puntos en la escala b de Minolta. El CENEB mantiene las categorías de semilla original y básica de esta variedad. La semilla registrada se ha incrementado a través del Patronato para la Investigación y Experimentación Agrícola en el Estado de Sonora (PIEAES). La siembra de 'Quetchehueca Oro C2013' se recomienda para el ciclo de producción otoño-invierno en la región del noroeste de México.

\section{AGRADECIMIENTOS}

A la Fundación Produce Sonora, A. C. (proyecto 9532632018) y al Patronato para la Investigación y Experimentación Agrícola en el Estado de Sonora (PIEAES), por el financiamiento parcial de los trabajos de investigación que condujeron a la obtención de la variedad 'Quetchehueca Oro C2013'. Al Centro Internacional de Mejoramiento de Maíz y Trigo (CIMMYT), por proporcionar las líneas avanzadas de donde se originó la variedad.

\section{BIBLIOGRAFÍA}

Camacho-Casas M. A., P. Figueroa-López y J. Huerta-Espino (2004) Júpare C2001, nueva variedad de trigo duro para su cultivo en el noroeste de México. Folleto Técnico No. 47. INIFAP-CIRNO, Campo Experimental Valle del Yaqui. Cd. Obregón, Sonora, México. 16 p.

CANIMOLT (Cámara Nacional de la Industria Molinera de Trigo) (2011) Reporte Estadístico al 2010. Julio 2011. Disponible en: http://www.canimolt.org/revista-canimolt. (Septiembre 2013).

FAOSTAT (Organización de las Naciones Unidas para la Alimentación y la Agricultura)(2011) World wheat production 2009. Disponible en: http://faostat.fao.org/site/567/DesktopDefault. aspx?PageID=567\#ancor. (Septiembre 2013).

INEGI (Instituto Nacional de Estadística y Geografía)(2010) Superficie agrícola sembrada, según cultivo y productos seleccionados. Disponible en: http://www.inegi.gob.mx/. (Septiembre 2013).

OEIDRUS (Oficina Estatal de Información para el Desarrollo Rural en Sonora) (2013) Concentrado Estatal. Ciclo Agrícola 20092010. Disponible en: http://www.oeidrus-sonora.gob.mx/. (Septiembre 2013).

Singh R. P., J. Huerta-Espino, W. Pfeiffer and L. P. Figueroa (2004) Ocurrence and impact of a new leaf rust race on durum wheat in the Northwestern Mexico during 2001-2002. Plant Disease 88:703-708. 


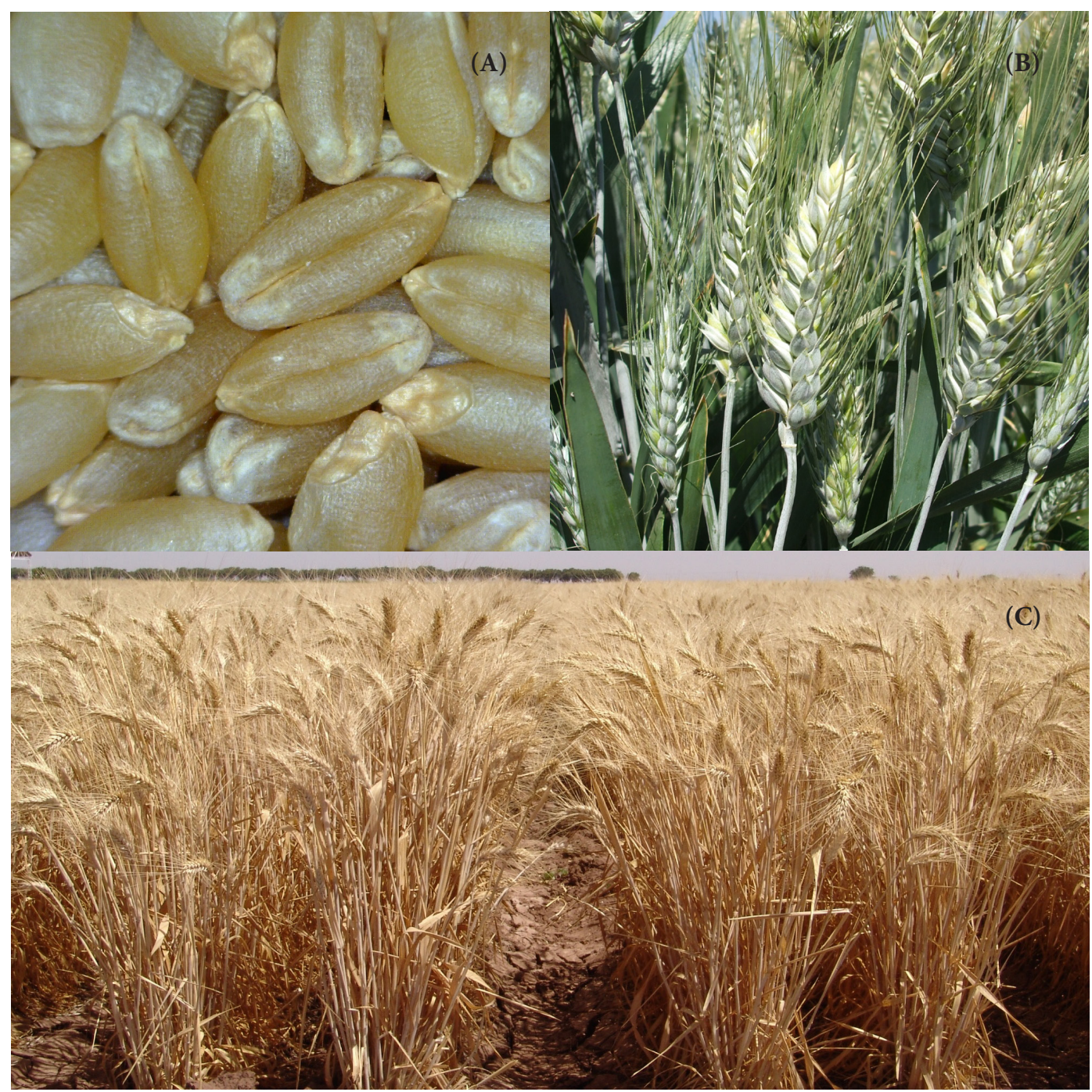

Figura 1. Aspecto de semillas (A) , espigas (B) , y planta en madurez (C) de la nueva variedad de trigo cristalino 'Quetchehueca Oro C2013', apropiada para el Noroeste de México. 